\title{
An Efficient Solver for the Stokes Equations with Random Inputs
}

\author{
Dong QU \\ College of Science, Jiujiang University \\ Jiangxi, China \\ E-mail: qudong_math@163.com
}

\author{
Jing YANG \\ College of Accounting, Jiujiang University \\ Jiangxi, China \\ E-mail: sixsun0924@sohu.com
}

\begin{abstract}
In this paper we present a high order method to solve the Stokes equations with random coefficients numerically. A stochastic Galerkin approach, based on the truncated KarhunenLoeve decomposition technique for the stochastic inputs, is used to reduce the original stochastic Stokes equations into a set of deterministic equations for the expansion coefficients. Then a spectral collocation method, together with a block Jacobi iteration is applied to solve the resulting problem. The efficiency of the solver is verified in each model problem by numerical tests, against Monte Carlo simulations.
\end{abstract}

Keywords-Random inputs; Wiener-Askey polynomial chaos; Spectral methods; Exponential error convergence

\section{INTRODUCTION}

Thanks to the great progress in the development of numerical methods and computer resources, many classical PDEs can now be solved very efficiently with high accuracy. However, in many cases, the inputs of the considered PDEs may contain uncertainties. In order to provide meaningful predictions to problems involving uncertain data, there is a need to investigate efficient numerical methods for handling general stochastic PDEs. In fact there exist several families of numerical techniques to treat such problems, e.g., Monte-Carlo simulations and its variants [1], perturbation methods via Taylor or Neumann expansions [2], etc.

In this paper, the stochastic Stokes equations describing the flow in a random medium determined by a random diffusion coefficient is considered. Firstly, the equation with random inputs is reduced to a finite-dimensional parametric saddlepoint problem by using a suitable Karhunen-Loeve decomposition with a suitable truncation. Secondly, we are focusing on its numerical solutions by using a method combining a stochastic projection method for the random variables and a classical spectral method [3] for the deterministic variables. Meanwhile, a block Jacobi iteration is applied in the implementation to decrease the computational cost, sine the resulting deterministic stokes-liking equations takes the strong coupling characters. Finally, numerical tests are illustrated to show the efficiency of the considered solver, against the Monte-Carlo simulations.

\section{PROBLEM DESCRIPTIONE}

We start by describing such a problem. Let $(\Omega, F, P)$ be a complete probability space. The stochastic Stokes problem considered here reads: Given $\mathbf{f} \in L^{2}(D)^{2}$, find the stochastic solution $\mathbf{u}(\mathbf{x}, \omega)$ and $p(\mathbf{x}, \omega)$ almost surely such that

$$
\left\{\begin{array}{c}
-\nabla \cdot(\kappa \nabla \mathbf{u})+\nabla p=\mathbf{f}(\mathbf{x}), \\
\operatorname{div} \mathbf{u}=0,
\end{array}\right.
$$

in $D \times \Omega$, together with a suitable boundary condition, e.g., $\mathbf{u}=\mathbf{0}$ on $\partial D \times \Omega$. Here, the diffusivity $\kappa(\mathbf{x}, \omega)$ is a stochastic function, which is assumed to have continuous and bounded correlation function.

Firstly, the probability space $\Omega$ should be reduced into a finite-dimensional space by some effective ways. That means the uncertainty needs to be characterized by a finite number of random variables through some effective ways, e.g., the truncated Karhunen-Loeve decomposition [4] once the correlation function of the stochastic input is known. After making the $K$-dimensional noise assumption, the random characters in $\kappa$ can be characterized by these $K$ random variables, and it can be rewritten as

$$
\kappa(\mathbf{x}, y)=\sum_{i=0}^{K} \kappa_{i}(\mathbf{x}) y_{i},
$$

where $y_{0}=1$, and $\left\{y_{k}\right\}_{k=1}^{K}$ are real random variables with zero mean and unite variance.

Following the Doob-Dynkin lemma, the solution $\mathbf{u}$ and $p$ can also be characterized by the same $K$ random variables such as

$$
\begin{aligned}
& \mathbf{u}(\mathbf{x}, \omega) \approx \mathbf{u}\left(\mathbf{x}, y_{1}, \cdots, y_{K}\right), \\
& p(\mathbf{x}, \omega) \approx p\left(\mathbf{x}, y_{1}, \cdots, y_{K}\right) .
\end{aligned}
$$

Furthermore, we also assume that $\left\{y_{k}\right\}_{k=1}^{K}$ are mutually independent random variables with probability density functions $\rho_{k}: \Gamma_{k} \rightarrow R^{+}$and their images $\Gamma_{k}$ are bounded intervals in $R$ for $k=1, \cdots, K$. Then $\rho(y)=\Pi_{k=1}^{K} \rho_{k}\left(y_{k}\right)$ is 
the joint probability density of $y=\left(y_{1}, \cdots, y_{K}\right)$ with the support $\Gamma \subset R^{K}$. This allows us to rewrite the stochastic stokes equation as a deterministic parametric saddle-point problem in the strong form, i.e.,

Find $\mathbf{u}=\mathbf{u}(\mathbf{x}, y)$ and $p=p(\mathbf{x}, y)$ satisfying

$$
\left\{\begin{array}{c}
-\nabla \cdot(\kappa \nabla \mathbf{u})+\nabla p=\mathbf{f}(\mathbf{x}), \\
\operatorname{div} \mathbf{u}=0
\end{array}\right.
$$

in $D \times \Gamma$, together with the corresponding boundary condition $\mathbf{u}=\mathbf{0}$ on $\partial D \times \Gamma$.

\section{WEAK FORMULATIONS}

In order to write out its corresponding weak formulation, some notations are introduced. we shall denote by $X=H_{0}^{1}(D)^{2}$ and $M=L_{0}^{2}(D)=\left\{q \in L^{2}(D), \int_{D} q=0\right\}$.

As to the stochastic aspects, the random fields will live in the tensor product of spaces defined on the spatial domain $D$, with spaces defined on the sample space $\Gamma$. For brief, we shall denote by

$$
X_{\rho}=X \otimes L_{\rho}^{2}(\Gamma) \text { and } M_{\rho}=M \otimes L_{\rho}^{2}(\Gamma) .
$$

Let $\kappa \in L^{\infty}(D) \otimes L^{\infty}(\Gamma)$. The weak formulation of the saddle-point problem (2) reads as follows:

Find $\mathbf{u}(\mathbf{x}, y) \in X_{\rho}, p(\mathbf{x}, y) \in M_{\rho}$, such that

$$
\left\{\begin{array}{c}
A(\mathbf{u}, \mathbf{v})-B(\mathbf{v}, p)=L(\mathbf{v}), \forall \mathbf{v} \in X_{\rho}, \\
B(\mathbf{u}, q)=0, \quad \forall q \in M_{\rho},
\end{array}\right.
$$

with

$$
\begin{gathered}
A(u, v)=\int_{\Gamma} \rho(\kappa \nabla u, \nabla v) d y, \forall u, v \in X_{\rho}, \\
B(v, q)=\int_{\Gamma} \rho(\operatorname{div} v, q) d y, \forall v \in X_{\rho}, q \in M_{\rho,} \\
L(v)=\int_{\Gamma} \rho(f, v) d y, \forall v \in X_{\rho} .
\end{gathered}
$$

Its well-posedness can be established in the framework for the abstract saddle-point problem [5]. Firstly, we have

$$
\inf _{q \in M_{\rho}} \sup _{v \in X_{\rho}} \frac{|B(v, q)|}{\|v\|_{X_{\rho}}\|q\|_{M \rho}} \geq \beta
$$

where $\beta$ is called the inf-sup constant. Finally, the weak formulation (3) can be verified that it admits one unique couple solution $(\mathbf{u}, p) \in X_{\rho} \otimes M_{\rho}$.

\section{DISCRETE FORMULATION AND IMPLEMENTATION}

\section{A. Discrete Formulation}

The polynomial chaos decomposition, as pioneered in [6], was generalized in [8] to solve PDEs with random data. It constructs a finite-dimensional subspace of $L_{\rho}^{2}(\Gamma)$, denoted by $Z_{K}^{P}(\Gamma)=\operatorname{span}\left\{\Phi_{0}, \cdots, \Phi_{Q}\right\}$, with $\left\{\Phi_{k}\right\}$ are WienerAskey orthogonal polynomial chaos with respect to $\rho(y)$, where $Q$ depends on $P$ and $K$, with $P$ is the highest order of the Wiener-Askey chaos. Note that if $\rho(y)$ is the density function corresponding to a $K$-dimensional Gaussian distribution, then $\left\{\Phi_{k}\right\}$ will be $K$-variate Hermite polynomials. If $\rho(y)$ is the density function corresponding to a $K$-dimensional uniform distribution, then $\left\{\Phi_{k}\right\}$ will be $K$ variate Legendre polynomials. For details of Wiener-Askey chaos, we refer to[8], etc.

The subspace $Z_{K}^{P}(\Gamma)$ can be constructed by a tensor product of one-dimensional subspace. It takes the form such as $Z_{K}^{P}(\Gamma)=\bigotimes_{k=1}^{K} Z_{1}^{P_{k}}\left(\Gamma_{k}\right)$, where $\sum_{k=1}^{K} P_{k}=P$, with the onedimensional global polynomial subspaces $Z_{1}^{P_{k}}\left(\Gamma_{k}\right)$ are defined by

$$
Z_{1}^{P_{k}}\left(\Gamma_{k}\right)=\operatorname{span}\left\{\phi_{0}\left(y_{k}\right), \cdots, \phi_{P_{k}}\left(y_{k}\right)\right\} \subset L_{\rho_{k}}^{2}\left(\Gamma_{k}\right),
$$

where $\phi_{n}\left(y_{k}\right)$ denotes the $n$-th order Wiener-Askey chaos with respect to univariate $y_{k}$, satisfies

$$
\int_{\Gamma_{k}} \rho_{k}\left(y_{k}\right) \phi_{i}\left(y_{k}\right) \phi_{j}\left(y_{k}\right) d y_{k}=\left\langle\phi_{i}^{2}\right\rangle \delta_{i j} .
$$

Denote by

$$
X_{N, P}=X_{N} \cap Z_{K}^{P}(\Gamma) \text {, and } M_{N, P}=M_{N} \cap Z_{K}^{P}(\Gamma)
$$

with $X_{N}, M_{N}$ are the Galerkin spectral approximation space [5] defined as $X_{N}=X \cap P_{N}(D)^{2}, M_{N}=M \cap P_{N}(D)$, where $P_{N}(D)$ denotes the polynomial spaces of degree less than $N$. Then the discrete formulation of (3) is as follows: Find $\mathbf{u}_{N, P} \in X_{N, P}, p_{N, P} \in M_{N, P}$ such that

$$
\left\{\begin{array}{c}
A\left(\mathbf{u}_{N, P}, \mathbf{v}_{N, P}\right)-B\left(\mathbf{v}_{N, P}, p_{N, P}\right)=L\left(\mathbf{v}_{N, P}\right), \\
B\left(\mathbf{u}_{N, P}, q_{N, P}\right)=0,
\end{array}\right.
$$

holds for each $\mathbf{v}_{N, P} \in X_{N, P}, q_{N, P} \in M_{N, P}$. Meanwhile, it can be verified that this discrete problem admits one unique solution couple $\left(\mathbf{u}_{N, P}, p_{N, P}\right) \in X_{N, P} \otimes M_{N, P}$.

\section{B. IMPLEMENTATION}

By applying $P$-th order Wiener-Askey polynomial chaos expansion to $\mathbf{u}$ and $p$, it yields

$$
\mathbf{u}(\mathbf{x}, y)=\sum_{i=0}^{Q} \mathbf{u}_{i}(\mathbf{x}) \Phi_{i}(y), p(\mathbf{x}, y)=\sum_{i=0}^{Q} p_{i}(\mathbf{x}) \Phi_{i}(y)
$$

with $\mathbf{u}_{i}(\mathbf{x})$ and $p_{i}(\mathbf{x})$ are deterministic functions. Substitute 
these above two expansions into the first equation of (2), and notice that the gradient operator $\nabla$ is taken just with respect to $\mathrm{x}$ and thus commutes with the operations in random space, the following equation can be achieved.

$$
-\sum_{j=0}^{Q} \nabla \cdot\left[\left(\sum_{i=0}^{K} \kappa_{i} y_{i} \Phi_{j}\right) \nabla \mathbf{u}_{j}\right]+\sum_{i=0}^{Q} \nabla p_{i} \Phi_{i}=\sum_{i=0}^{Q} \mathbf{f}_{i} \Phi_{i},
$$

Multiplying the above equation by $\left\{\Phi_{k}\right\}$ on both sides and taking the mean value, together with the orthogonality of $\left\{\Phi_{k}\right\}$, thus for each $k=0,1, \cdots, Q$, it holds that

$$
-\sum_{j=0}^{Q} \nabla \cdot\left[s_{j k}(\mathbf{x}) \nabla \mathbf{u}_{j}(\mathbf{x})\right]+\left\langle\Phi_{k}^{2}\right\rangle \nabla p_{k}(\mathbf{x})=\left\langle\Phi_{k}^{2}\right\rangle \mathbf{f}_{k}(\mathbf{x}),
$$

where $S_{j k}(\mathbf{x})=\sum_{i=0}^{Q} \kappa_{i}(\mathbf{x}) M_{i j k}, M_{i j k}=\left\langle y_{i} \Phi_{j} \Phi_{k}\right\rangle$.

Together with $\left\langle\Phi_{k}^{2}\right\rangle$, the coefficient $M_{i j k}$ can be evaluated analytically from the definition of $\left\{\Phi_{k}\right\}$. A similar treatment for the other two equations of (2) yields

$$
\begin{aligned}
& \nabla \cdot \mathbf{u}_{k}(\mathbf{x})=0, \forall \mathbf{x} \in D ; \\
& \mathbf{u}_{k}(\mathbf{x})=\mathbf{0}, \forall \mathbf{x} \in \partial D
\end{aligned}
$$

The system (4)-(5) is a set of $Q+1$ coupled Stokes-liking problem, which governs the evolution of chaos expansion coefficients $\left\{\mathbf{u}_{k}, p_{k}\right\}_{k=0}^{Q}$. These equations are deterministic and can be solved by any conventional method. In this paper, the $\mathrm{P}_{N} \times \mathrm{P}_{N-2}$ spectral Galerkin method together with Uzawa techniques [5] is used. While it is possible to solve (7)-(8) via a direct solver, the block Jacobi iteration [7] is employed which takes the form: for $k=0,1, \cdots, Q$, solving

$$
\left\{\begin{array}{c}
-\nabla \cdot\left(s_{k k} \nabla \mathbf{u}_{k}^{(n+1)}\right)+\left\langle\Phi_{k}^{2}\right\rangle \nabla p_{k}^{(n+1)}=\left\langle\Phi_{k}^{2}\right\rangle \mathbf{f}_{k}+\sum_{i \neq k} \nabla \cdot\left(s_{k i} \nabla \mathbf{u}_{i}^{(n)}\right), \\
\nabla \cdot \mathbf{u}_{k}^{(n+1)}=0, \forall \mathbf{x} \in D, \\
\mathbf{u}_{k}^{(n+1)}=0, \forall \mathbf{x} \in \partial D,
\end{array}\right.
$$

where the subscript $(n)$ denotes the iteration count. The convergence criterion is defined as

$$
\max _{k}\left\{\frac{\left\|\mathbf{u}_{k}^{(n+1)}-\mathbf{u}_{k}^{(n)}\right\|}{\left\|\mathbf{u}_{k}^{(1)}-\mathbf{u}_{k}^{(0)}\right\|}, \frac{\left\|p_{k}^{(n+1)}-p_{k}^{(n)}\right\|}{\left\|p_{k}^{(1)}-p_{k}^{(0)}\right\|}\right\} \leq \varepsilon,
$$

where $\varepsilon$ is a small positive number and different type of norm $\|\cdot\|$ can be used. In this paper the $L^{\infty}(D)$ norm is used and $\varepsilon$ is set to be $10^{-10}$.

\section{NUMERICAL TEST}

To demonstrate the solver's convergence character, a model with the available exact solution will be considered firstly.
Finally, a stochastic Stokes problem, in which $\kappa$ and $f$ are assumed to be stochastic functions with an exponential correlation function, is examined as an example of more complicated practical applications.

In all models, let $D=[-1,1]^{2}$, and $N=16$ is chosen in the spectral Galerkin method to avoid the influence of spatial approximations to the stochastic aspects. Moreover, $\left\{y_{k}\right\}_{k=1}^{K}$ are also prescribed to be uniform random variables in $\Gamma_{k}=[-1,1]$, so the corresponding optimal chaos is still the Legendre-chaos.

Test 1. Let $\kappa\left(\mathbf{x}, y_{1}\right)=1+\cos y_{1}$. Consider the Stokes problem with the exact solution such as

$$
\begin{aligned}
& \mathbf{u}=\left(\begin{array}{c}
\sin \left(\pi x_{1}\right) \cos \left(\pi x_{2}\right)\left(1+\sin y_{1}\right) \\
-\cos \left(\pi x_{1}\right) \sin \left(\pi x_{2}\right)\left(1+\sin y_{1}\right)
\end{array}\right), \\
& p=\sin \left(\pi x_{1}\right) \cos \left(\pi x_{2}\right)\left(1+\sin y_{1}\right) .
\end{aligned}
$$

Here, different values for $P$ is examined in the process of computations. Results show that the block Jacobi iterative scheme converges at most 3 iterations for each computation. Figure 1 shows the convergence rate of the approximation error with respect to the order $P$ used in the Legendre-chaos expansion. Since the logarithm arithmetic has been done for the coordinate which characterizes the error property, the exponential rate of convergence is achieved.

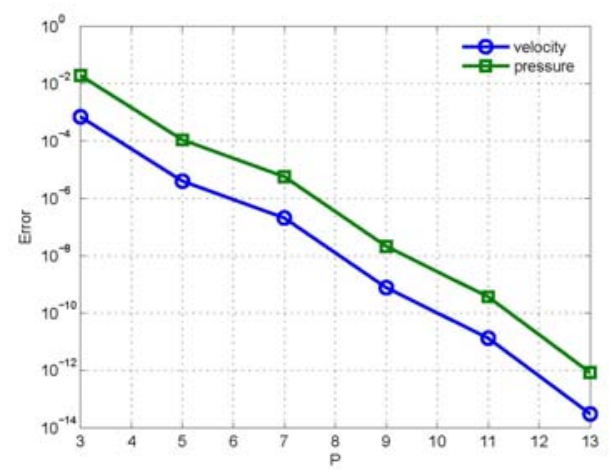

Figure 1 . The convergence character for the Legender-chaos decomposition.

Test 2. A general case that both $\kappa$ and $f$ are stochastic processes is examined. Here, the exponential correlation function $C(\mathbf{x}, \mathbf{z})=\exp \left(-\frac{|\mathbf{x}-\mathbf{z}|}{b}\right)$ is employed. Due to the fast decay of its eigenvalues with $b=100$ (see Figure 2), the fourth order truncation is taken in the KL decomposition. This results in a four-dimensional (in random space) Legendrechaos decomposition. Here, we set $\langle\kappa\rangle=4$, and

$$
\langle\mathbf{f}\rangle=\left(\begin{array}{c}
8 \pi^{2} \sin \left(\pi x_{1}\right) \cos \left(\pi x_{2}\right)+\pi \cos \left(\pi x_{1}\right) \cos \left(\pi x_{2}\right) \\
-8 \pi^{2} \cos \left(\pi x_{1}\right) \sin \left(\pi x_{2}\right)-\pi \sin \left(\pi x_{1}\right) \sin \left(\pi x_{2}\right)
\end{array}\right) .
$$


Resolution checks in stochastic space were conducted, and it was shown that third-order $(P=3)$ Legendre-chaos results in converged solution. For four-dimensional Legendre-chaos ( $K=4$ ), the total number of decomposition terms is 35 . In figure 3 , the convergence character of the block Jacobi iterative scheme is shown.

Since no analytical solution is available, the Monte Carlo simulation is employed to validate the Legendre-chaos solution. Here, the Monte Carlo computation is employed after the same KL decomposition. In this way the error from the Legendre-chaos decomposition is isolated, while the error introduced by the finite-term truncation of the $\mathrm{KL}$ decomposition, which is well understood, is excluded. The corresponding computational results associated with the $L^{2}(D)^{2} \otimes L^{2}(\Gamma)$ norm has been shown in Figure 4.

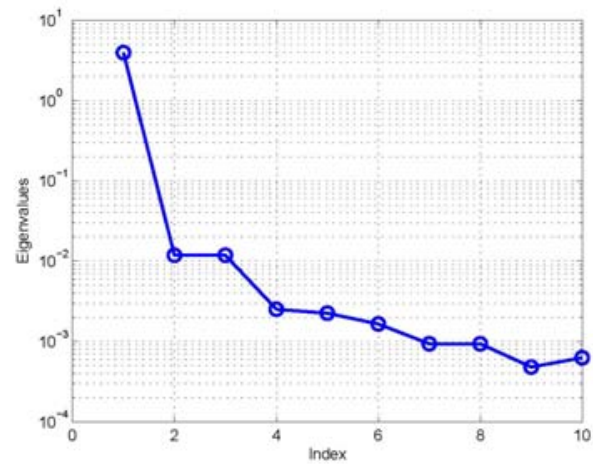

Figuer 2. Eigenvalues of KL decompositon for $C(\mathbf{x}, \mathbf{z})$ with $b=100$.

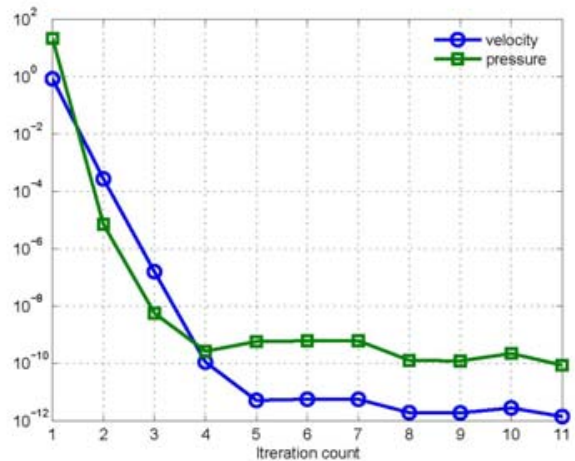

Figure 3. Convergence of error norm in term of iteration counts for the block Jacobi iterative scheme with $K=4, P=3$.

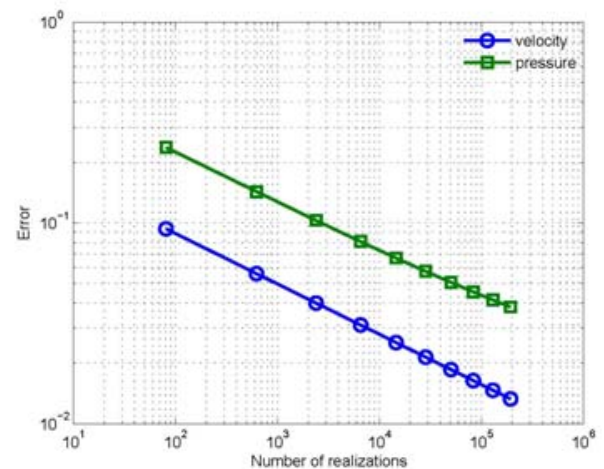

Figure 4. Comparison between the third-order Legendre-chaos solution and the Monte Carlo solution with various number of realizations.

\section{CONCLUSION}

In this paper, we have presented a stochastic spectral method to the Stokes problems with random inputs. The essential finite dimensional noise assumption turns the original Stokes problem into a parametric saddle-point problem. It was solved by using the generalized polynomial chaos decomposition, together with a spectral Galerkin approximation in the spatial domain. We have employed a block Jacobi iteration technique to solve the system which governs the evolution of the chaos decomposition coefficients efficiently. The exponential correlation function was studied and applied in the computations. The Karhunen-Loeve decomposition is used to reduce the dimensionality of the stochastic space.

The exponential convergence rate is demonstrated for the model problem with $K=1$. For more complicated Stokes problems, the Monte Carlo simulation is employed to validate the chaos solution. We have observed good agreement between the well-resolved chaos decomposition solution and the converged Monte Carlo simulation results.

\section{REFERENCES}

[1] G.S. Fishman, Monte Carlo: Concepts, Algorithms, and Applications, Springer-Verlag, New York, Inc., 1996.

[2] R.G. Ghanem and P. Spanos, Stochastic Finite Elements: A Spectral Approach, Springer-Verlag, 1991. [Reedited by Dover Publications, 2003.]

[3] Canuto, M.Y. Hussaini, A. Quarteroni and T.A. Zang, Spectral method in fluid dynamics, Springer-Verlag, New York, 1988.

[4] M. Loeve, Probability Theory, I. 4th ed., Grad. Texts in Math. 45, Springer-Verlag, New York, 1977.

[5] Quarteroni and A. Valli, Numerical Approximations of Partial Differential Equations, Springer, 1994.

[6] N. Wiener, The homogenous chaos, Amer. J. Math., 60:897-936, 1938.

[7] Y. Saad, Iterative Methods for Sparse Linear Systems, Society for Industrial Mathematics, 2003.

[8] Xiu and G. Karniadakis, The Wiener-Askey Polynomial Chaos for Stochastic Differential Equations, SIAM Journal on Scientific Computing,24(2):619-644,2002. 